\title{
Software Development for Automated Network Design Supporting Unicast and Multicast Traffics in Next Generation Network
}

\author{
Kalika Suksomboon \\ kmitmink@yahoo.com
}

\author{
Piyanan Satayapiwat \\ piyanan.satayapiwat \\ @gmail.com
}

\author{
Chaodit Aswakul \\ chaodit.a@chula.ac.th
}

Department of Electrical Engineering, Faculty of Engineering, Chulalongkorn University, Payathai Rd., Pathumwan, Bangkok 10330, Thailand

\begin{abstract}
This paper presents a summary of our experiences in developing a software tool for IP/MPLS NGN traffic engineering computations. The developed NGN software consists of three main modules. Firstly, the NGN design module can be used to help network engineers find the minimum link capacity needed for NGN unicast (e.g. VoIP service) and multicast service (e.g. VDO conference) provision while maintaining the resultant QoS at a specified target level. Secondly, the NGN performance analysis module is based on a discrete-event simulation of sequential routing and trunk reservation CAC. This module can be used to help network engineers analyse the performance of NGN. It can also be used to predict the effect of link failure, traffic surge and new routing plan implementation. Finally, both the NGN design and performance analysis modules can be executed via the developed NGN GUI module. With GUI, network engineers can visualise the status of network components. Further, GUI permits both entering and editing all relevant network parameters efficiently. It is therefore very convenient for our developed NGN software to be utilised in practice and should be an indispensable traffic engineering tool for improving the NGN planning and operational tasks.
\end{abstract}

\section{Categories and Subject Descriptors}

C.2.1 [Computer Systems Organization]: Computer-Communication Networks,Network Architecture and Design

\section{Keywords}

NGN software, Network design, Network performance, GUI

\section{INTRODUCTION}

${ }^{1}$ At the rise of telecommunication needs, telecommunication markets worldwide have experienced greater transformations than ever before. The service provision paradigm in many countries has changed from the monopoly by state-own organisations into the market with many competing providers. In order to survive, each service provider must try to do their best by minimising service

\footnotetext{
${ }^{1}$ Permission to make digital or hard copies of all or part of this work for personal or classroom use is granted without fee provided that copies are not made or distributed for profit or commercial advantage and that copies bear this notice and the full citation on the first page. To copy otherwise, or republish, to post on servers or to redistribute to lists, requires prior specific permission and/or a fee.

SIMUTools, March 03 - 07, 2008, Marseille, France, ISBN 978-963-979920-2
}

provision costs while maintaining the acceptable level for quality of service (QoS). Such attempts must be seriously considered throughout the whole period of network lifetime, starting from its inception planning stage towards the actual implementation as well as the network employment and maintenance stages.

Apart from the change in market structure, many new networking technologies have in the past decade been invented. Nowadays, it is clear that both legacy and contemporary telecommunication platforms are planned along various roadmaps with the same goal towards the Internet Protocol (IP) [1]. Core network services are envisioned to be all-IP and achievable IP QoS is facilitated by the traffic engineering capabilities of multi-protocol label switching (MPLS) network. This IP/MPLS suite becomes the principle part in the next generation network (NGN) [2].

From network engineering viewpoint, IP/MPLS NGN provides many technical advantages, e.g. lowering the operational cost from the economy of scales while providing a common platform flexible enough for initiation of future new services. In practice, to help engineers implement NGN, there exist many off-the-shelf softwares (e.g. [3], [4]). However, these software have been developed as a general platform that is applicable to a wide range of network technologies. Such general-purpose software is typically expensive, highly complicated and hence usually cannot be afforded by both academia and engineering practitioners in developing countries.

To overcome these drawbacks, we have chosen to develop our own software specifically optimised for IP/MPLS NGN. Further, the developed NGN software must be able to execute an automatic network design procedure to help engineers in network planning stage. In this respect, we have formulated a mathematical algorithm to design network in both cases of unicast and multicast traffics. The objective is to minimise the overall network cost to support a given traffic demand at the desired QoS level. Moreover, in the network employment stage, the NGN software can be applied to identify network bottlenecks and predict the results that can be expected upon different candidate network upgrading solutions via computer simulation embedded in the software. This paper is intended as a summary report of our experiences in developing this software, which is co-designed by academic researchers and the practical network engineers in the industry [5]. For educational purposes, our developed software is made available at http://pioneer.netserv.chula.ac.th/ achaodit.

The remainder of paper is organised as follows. In Section 2, based on the framework of traffic engineering, the overall architecture of NGN software is introduced. Section 3 gives the mathematical formulation of automatic NGN design. Section 4 explains 


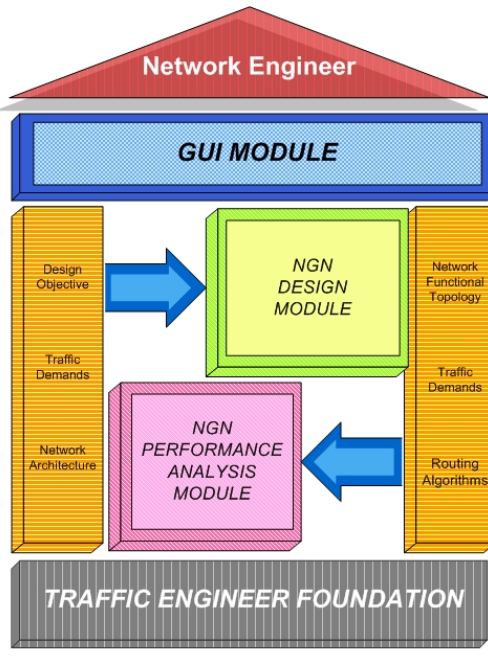

Figure 1: Architecture of the NGN software.

how QoS parameters of NGN can be measured in the developed discrete-event computer simulation. Numerical examples obtained from the software are given in Section 5. Finally, in Section 6, a summary of achievements relevant to this software development are given.

\section{NGN SOFTWARE ARCHITECTURE}

Figs. 1 and 2 depict the overall architecture and data flow diagram of implemented NGN software. This software has been designed on the foundation of traffic engineering. It consists of three main modules, namely, NGN design module, NGN performance analysis module and GUI module. Network engineer can execute NGN design module in mapping from input design objectives, traffic demands and underlying network architecture (e.g. topology and link cost matrix) into a functional network topology with assigned link capacity matrix and a minimum-cost routing plan. Further, depending on different routing algorithms being actually employed in NGN, engineer can also use NGN performance analysis module to simulate how traffic demands would be distributed across route candidates and how such distribution affects the link utilisation as well as resultant QoS parameters. All input and output parameters can be interfaced via the GUI, which has been specifically designed in our software based on feedbacks from practical network engineers who have experimented up with our software. Therefore, GUI in our software can be easy to use and understand by those directly responsible for planning the real NGN platforms.

\section{MATHEMATICAL FORMULATION OF NGN DESIGN}

\subsection{Design Objective and Assumptions}

In the NGN software, the design objective is to minimise the overall network cost needed to provide a specified set of traffic demands with their desired QoS targets. These QoS targets are classified into two levels of time scale.

- Level of connection or call dynamics i.e. call blocking probability and average call setup delay.

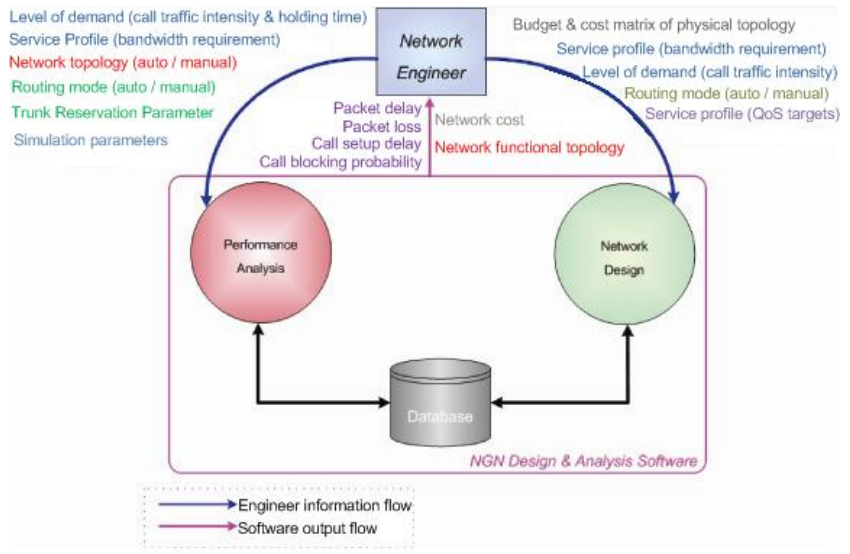

Figure 2: Data flow diagram of the NGN software.

- Level of packet dynamics i.e. packet loss probability and average packet delay.

Naturally different services require different levels of these QoS targets. Therefore, it is unwise to allow packet streams from different services to statically share the same portion of network resources. By doing so, it would then require that engineers must design that portion of network to satisfy the service with the most stringent target on QoS. As a result, all the other traffic streams from lower-quality service would be treated too well, which causes inefficiency in utilising network resources. For this reason, in the NGN software, we assume that each service must have its own virtual private network (VPN) [13] and each VPN is allocated a fixed amount of capacity, which is not shared by other VPNs. This mechanism is known as complete partitioning scheme and has been thoroughly analysed in the past (e.g. [6]).

Another main advantage of complete partitioning scheme is that it simplifies the bandwidth dimensioning problem a great deal. In this regard, we have adopted the well-known notion of equivalent capacity function (e.g. [7]). This function defines the mapping function from the number of connections into the required link capacity to guarantee that all the connections will meet their designed QoS targets.

For the algorithm explanation in this paper, all demands are classified into two types i.e. unicast type and multicast type. For the unicast type of demands, each connection is terminated between a pair of originating and destination nodes. The multicast type, however, differs from the unicast type of demands in that a source node spreads all traffics towards a group of destination nodes. The implemented NGN software can also be applied to capture other types of services equally well (e.g. multiple-unicast IP Centrex or IP PABX service, unicast data service, unicast signalling service and multicast VDO conference).

\subsection{Automatic NGN Design Algorithm for Uni- cast Traffics}

The NGN design module first calculates the minimum cost routing plan for each origin-destination node pair by the well-known Dijkstra algorithm [8]. Here, let $r_{O D}$ denote the set of links along the minimum-cost path from origin node $O$ to destination node $D$. Also, let

$$
\delta\left(l, r_{O D}\right)= \begin{cases}1, & l \in r_{O D} \\ 0, & \text { otherwise }\end{cases}
$$


and define the number of links on route $r_{O D}$ as

$$
L\left(r_{O D}\right)=\sum_{l} \delta\left(l, r_{O D}\right)
$$

Let the call traffic intensity between node pair $O D$ of the demand service category $s$ be denoted as $d_{O D}(s)$. This demand requires the call blocking probability $C B P_{O D}(s)$ not greater than target value $C B P_{t}(s)$; the packet loss probability $P L_{O D}(s)$ not greater than target value $P L_{t}(s)$; and the average packet delay $P D_{O D}(s)$ not greater than target value $P D_{t}(s)$. Note that the average call setup delay is herein not considered because it needs a special attention and all signalling traffics would be sent via a dedicated signalling VPN. For the design of a service VPN, the aim is to find the minimum cost $M^{*}(s)$ of building a VPN for demand type $s$ and the capacity of each link $l, y_{l}^{*}(s)$ that can satisfy the QoS needs on the VPN. VPN design process for this point-to-point unicast service is explained in the following steps.

\subsubsection{Computation of the call traffic intensity offered to each link $l$}

The total demand offered to a link is obtained from a direct summation of all individual demands passing through that link. This is based on the standard assumption that each demand follows an independent Poisson process.

$$
f_{l}(s)=\sum_{O D} d_{O D}(s) \delta\left(l, r_{O D}\right)
$$

\subsubsection{Computation of call blocking probability}

Assume for calls between each node pair that call blocking occurs independently and identically on all the links along the minimumcost path. The design constraint on call blocking probability on each origin-destination pair can then be written as

$$
\prod_{l \in r_{O D}}\left[1-C B P_{l}(s)\right]=\left[1-C B P_{l}(s)\right]^{L\left(r_{O D}\right)} \geq 1-C B P_{t}(s)
$$

where $C B P_{l}(s)$ is the call blocking probability of demand type $s$ on link $l$. To satisfy this design constraint, we choose the appropriate value $C B P_{l}^{*}(s)$ according to the demand that passes through link $l$ along the longest path (with the number of hops equal to $\left.\max _{O D} L\left(r_{O D}\right)\right)$. It follows that

$$
C B P_{l}^{*}(s)=1-\left[1-C B P_{t}(s)\right]^{1 / \max \left(L\left(r_{O D}\right)\right)}
$$

Finally, we can calculate the call blocking probability of demand type $s$ between node pair $O D$

$$
C B P_{O D}(s)=1-\prod_{l \in r_{O D}}\left(1-C B P_{l}^{*}(s)\right)
$$

and the overall call blocking probability from this automatic design algorithm

$$
C B P_{d}(s)=\frac{\sum_{O D} d_{O D}(s) C B P_{O D}(s)}{\sum_{O D} d_{O D}(s)}
$$

\subsubsection{Computation of packet loss probability}

Similar to the call blocking computation, we assume that packet loss occurs independently and identically on all the traversing links of each minimum-cost path. It follows in the similar manner to (2) and (3) that the chosen value for packet loss probability on link $l$ for demand $s$

$$
P L_{l}^{*}(s)=1-\left[1-P L_{t}(s)\right]^{1 / \max \left(L\left(r_{O D}\right)\right)}
$$

and the packet loss probability of demand $s$ between node pair $O D$

$$
P L_{O D}(s)=1-\prod_{l \in r_{O D}}\left(1-P L_{l}^{*}(s)\right)
$$

Finally, the overall packet loss probability from this automatic design algorithm can be obtained from

$$
P L_{d}(s)=\frac{\sum_{O D} P L_{O D}(s) d_{O D}(s)\left(1-C B P_{O D}(s)\right)}{\sum_{O D} d_{O D}(s)\left(1-C B P_{O D}(s)\right)}
$$

\subsubsection{Computation of required number of channels}

Knowing the call offered traffic $f_{l}(s)$ and the number of channels $z_{l}(s)$, we can calculate the resultant call blocking probability $C B P_{l}(s)$ straightforwardly from the Erlang loss function

$$
\begin{aligned}
C B P_{l}(s) & =\operatorname{Erlang}\left(f_{l}(s), z_{l}(s)\right) \\
& =\frac{\left[f_{l}(s)\right]^{z_{l}(s)} /\left[z_{l}(s)\right] !}{\sum_{i=0}^{z_{l}(s)}\left[f_{l}(s)\right]^{i} / i !}
\end{aligned}
$$

In the NGN design module, we take the inverse of this Erlang loss function and the number of channels $z_{l}^{*}(s)$ required on link $l$ for demand $s$ can be computed from

$$
z_{l}^{*}(s)=\operatorname{Erlang}^{-1}\left(C B P_{l}^{*}(s), f_{l}(s)\right)
$$

\subsubsection{Computation of link capacity}

In the typical case of circuit switching, knowing the number of channels is sufficient to determine the required link capacity because they are directly proportional to each other. However, in IPbased NGN, packet streams from connections sharing the same link can share the capacity of that link in a statistical manner. This is the so-called statistical multiplexing and its gain allows the economy of scales. The more connections are sharing a link, the less capacity on that link is required per connection. In order to capture this statistical multiplexing effect, we adopt the standard notion of equivalent capacity function [7]. That is, we can calculate the link capacity from

$$
\begin{gathered}
y_{l}^{*}(s)=\min \left\{m+\alpha^{\prime} \sigma, z_{l}^{*}(s) \hat{c}\right\} \\
m=R_{\text {peak }} \rho z_{l}^{*}(s) \\
\alpha^{\prime}=\sqrt{-2 \ln \left(P L_{l}^{*}(s)\right)-\ln (2 \pi)} \\
\sigma=R_{\text {peak }} \sqrt{\rho(1-\rho) z_{l}^{*}(s)} \\
\hat{c}=\left\{\sqrt{\left[\alpha b(1-\rho) R_{\text {peak }}-x\right]^{2}+4 x \alpha b \rho(1-\rho) R_{\text {peak }}}\right. \\
\left.+\alpha b(1-\rho) R_{\text {peak }}-x\right\} /[2 \alpha b(1-\rho)] \\
\alpha=\ln \left(1 / P L_{l}^{*}(s)\right)
\end{gathered}
$$

where $\left(b, \rho, R_{\text {peak }}\right)$ is the mean burst period, source utilisation and peak bit rate of each connection. These parameters $\left(b, \rho, R_{\text {peak }}\right)$ depend mainly on the type of service and their standard values are also given as defaults in the NGN software. Further, $x$ is the size of buffer (packets). In practice, $x$ must be chosen appropriately such that the average packet delay satisfies its target. To that respect, network engineers can try on various buffer size configurations and iteratively execute the NGN design software to automatically calculate the resulting average packet delay until the average packet delay target is satisfied. 


\subsubsection{Computation of packet delay bound}

The packet delay for demand type $s$ between node pair $O D$ is upper-bounded by

$$
P D_{O D}(s) \leq \sum_{l \in r_{O D}}\left[\frac{P S_{a}(s)+x}{y_{l}^{*}(s)}\right]
$$

where $P S_{a}(s)$ denote the average packet size of demand $s$. The packet delay for all node pairs of demand type $s$ is finally obtained from

$$
P D_{d}(s)=\frac{\sum_{O D} P D_{O D}(s) d_{O D}(s)\left(1-C B P_{O D}(s)\right)}{\sum_{O D} d_{O D}(s)\left(1-C B P_{O D}(s)\right)}
$$

\subsubsection{Computation of network cost}

Given $C_{l}$ is the cost per capacity unit of link $l$, we can compute for the cost of building VPN for demand type $s$

$$
M^{*}(s)=\sum_{l} y_{l}^{*}(s) C_{l}
$$

Note that, due to the complete partitioning scheme, the total cost of building multiple VPNs for supporting all VPNs of demand type $s$

$$
M^{*}=\sum_{j} M^{*}(s)
$$

where $j$ is the index of VPN.

\subsection{Automatic NGN Design Algorithm for Mul- ticast Traffics}

The NGN design module first computes a minimum spanning tree (MST) to connect a source to its group of destinations for each multicast session. To connect each source and all its destinations with the minimum cost, we adopt the MST algorithm based on genetic algorithm (GA) [14]. The muticast routing with MST $r_{k}$ is obtained for multicast session $k$, which consists of the tuples (OD) of common origin $O$ and all its corresponding destinations $D$, collectively represented by $O D \in \Upsilon_{k}$. Also, let the route between origin $O$ and destination $D$ in the assigned MST $r_{k}$ be denoted as $r_{O D, k}$. Here, let

$$
\delta\left(l, r_{k}\right)= \begin{cases}1, & l \in r_{k} \\ 0, & \text { otherwise }\end{cases}
$$

and

$$
\gamma\left(l, r_{O D, k}\right)= \begin{cases}1, & l \in r_{O D, k} \\ 0, & \text { otherwise }\end{cases}
$$

The number of hops along route $r_{O D, k}$, denoted as $L\left(r_{O D, k}\right)$, can be calculated from

$$
L\left(r_{O D, k}\right)=\sum_{l} \gamma\left(l, r_{O D, k}\right)
$$

The definitions of the other parameters are the same as the unicast traffic case. VPN design process for a point-to-multipoints multicast service is explained in the following steps.

\subsubsection{Computation of the call traffic intensity offered to each link $l$}

We assume that a VPN supporting multicast application of service category $s$ contains several multicast sessions. Each session contains traffic demand with the same QoS target values. The total demand in a VPN is the summation of demands from all multicast sessions in that VPN. Therefore, the total demand offered to a link is obtained from a direct summation of all individual demands passing through that link

$$
f_{l}(s)=\sum_{k} d_{k}(s) \delta\left(l, r_{k}\right)
$$

where $d_{k}(s)$ is the call traffic intensity of multicast session $k$ in service category $s$.

\subsubsection{Computation of call blocking probability}

In this work, it is assumed for call between each node pair in every multicast session that call blocking occurs independently and identically on all links. The calculation of call blocking probability value satisfying the call blocking probability target constraint can be written as

$$
C B P_{l}^{*}(s)=1-\left[1-C B P_{t}(s)\right]^{1 / \max _{k}\left(\max _{O \in \Upsilon_{k}} L\left(r_{O D, k}\right)\right)}
$$

where $C B P_{l}^{*}(s)$ is chosen according to demand type $s$ that passes through link $l$ along the longest path in the group of multicast sessions (with the number of total hops equal $\max _{k}\left(\max _{O D} L\left(r_{O D, k}\right)\right)$ ). The call blocking probability of demand type $s$ between node pair $O D \in \Upsilon_{k}$ can be calculated from

$$
C B P_{O D}(s)=1-\prod_{l \in r_{O D, k}}\left(1-C B P_{l}^{*}(s)\right)
$$

and the overall call blocking probability of session $k$ is

$$
C B P_{k}(s)=\max _{O D \in \Upsilon_{k}} C B P_{O D}(s)
$$

Let a multicast VPN with multiple multicast sessions carrying traffic type $s$ be indexed by $j$ and the call blocking probability of VPN $j$ be denoted as $C B P_{V P N_{j}}$. Finally, $C B P_{V P N_{j}}$ can then be calculated from the automatic design algorithm

$$
C B P_{V P N_{j}}(s)=\frac{\sum_{k \in V P N_{j}} d_{k}(s) C B P_{k}(s)}{\sum_{k \in V P N_{j}} d_{k}(s)}
$$

\subsubsection{Computation of packet loss probability}

Similar to the call blocking probability computation in multicast traffic case, it is assumed that packet loss occurs independently and identically on all the traversing links of minimum-cost tree. It follows that the computation process of packet loss probability is similar to (15) and (16). The selected value of packet loss probability on link $l$ for demand type $s$ is directly computed from

$$
P L_{l}^{*}(s)=1-\left[1-P L_{t}(s)\right]^{1 / \max _{k}\left(\max _{O \in \Upsilon_{k}} L\left(r_{O D, k}\right)\right)}
$$

and the packet loss probability between node pair OD is

$$
P L_{O D}(s)=1-\prod_{l \in r_{O D, k}}\left(1-P L_{l}^{*}(s)\right)
$$

The packet loss probability of session $k$ can be obtained from

$$
P L_{k}(s)=\frac{\sum_{O D \in \Upsilon_{k}} P L_{O D}(s) d_{O D}(s)\left(1-C B P_{k}(s)\right)}{\sum_{O D \in \Upsilon_{k}} d_{O D}(s)\left(1-C B P_{k}(s)\right)}
$$

where $d_{O D}(s)$ is the demand between node pair OD in the session. Since every node pair OD has equal call traffic intensity within the same multicast session $\left(d_{O D}(s)=d_{k}(s), \forall O D \in k\right)$, (21) can 
further be reduced to

$$
P L_{k}(s)=\frac{\sum_{D \in \Upsilon_{k}} P L_{O D}(s)\left(1-C B P_{k}(s)\right)}{\sum_{D \in \Upsilon_{k}}\left(1-C B P_{k}(s)\right)}
$$

Finally, let $P L_{V P N_{j}}(s)$ denote the overall packet loss probability of VPN $j$ containing all multicast sessions $k \in V P N_{j}$. The automatic design algorithm calculates $P L_{V P N_{j}}(s)$ from

$$
P L_{V P N_{j}}(s)=\frac{\sum_{k \in V P N_{j}} P L_{k}(s) d_{k}(s)\left(1-C B P_{k}(s)\right)}{\sum_{k \in V P N_{j}} d_{k}(s)\left(1-C B P_{k}(s)\right)}
$$

\subsubsection{Computation of required number of channels and link capacity}

The computation process of required number of channels and link capacity for the multicast traffic are the same as the computation for the unicast traffic. After the call offered traffic $f_{l}(s)$ and the call blocking probability of links $C B P_{l}^{*}(s)$ are known, the number of required channels can be computed from the inverse Erlang loss function (8). Subsequently, given all the input traffic parameters, the appropriate amount of link capacity can then be assigned to all links using the equivalent capacity function (9).

\subsubsection{Computation of packet delay bound}

Similar to the computation of packet loss for the multicast traffic case, the packet delay of demand $s$ between node pair OD can be upper-bounded by

$$
P D_{O D}(s) \leq \sum_{l \in r_{O D, k}}\left[\frac{P S_{a}(s)+x}{y_{l}^{*}(s)}\right]
$$

and the packet delay for multicast session $k$ is obtained from

$$
P D_{k}(s)=\frac{\sum_{O \Upsilon_{k}} P D_{O D}(s)\left(1-C B P_{k}(s)\right)}{\sum_{O D \in \Upsilon_{k}}\left(1-C B P_{k}(s)\right)}
$$

Let the overall packet delay of VPN $j$ be denoted as $P D_{V P N_{j}}(s)$. $P D_{V P N_{j}}(s)$ is computed from

$$
P D_{V P N_{j}}(s)=\frac{\sum_{k \in V P N_{j}} P D_{k}(s) d_{k}(s)\left(1-C B P_{k}(s)\right)}{\sum_{k \in V P N_{j}} d_{k}(s)\left(1-C B P_{k}(s)\right)}
$$

\subsubsection{Computation of network cost}

Similar to the unicast traffic case, we can compute the cost of building VPN for demand type $s$ from (12). Furthermore, the total cost of building all VPNs of service type $s$ can then be calculated by (13).

\section{PERFORMANCE ANALYSIS OF NGN}

The outputs from the NGN design module are the VPN topology, link capacity and resultant QoS levels. Since the network design is an iterative process, the NGN design module has been implemented with simplifications to accelerate the computational time. Unlike the NGN design module, the NGN performance analysis module is used to simulate the actual employment of the design network. The essential aim is to be a comprehensive guide to evaluate the network performance. In the real practice, an alternative route is assigned to a call whose main route experiences a congestion. For this reason, in order to capture this alternative routing, the

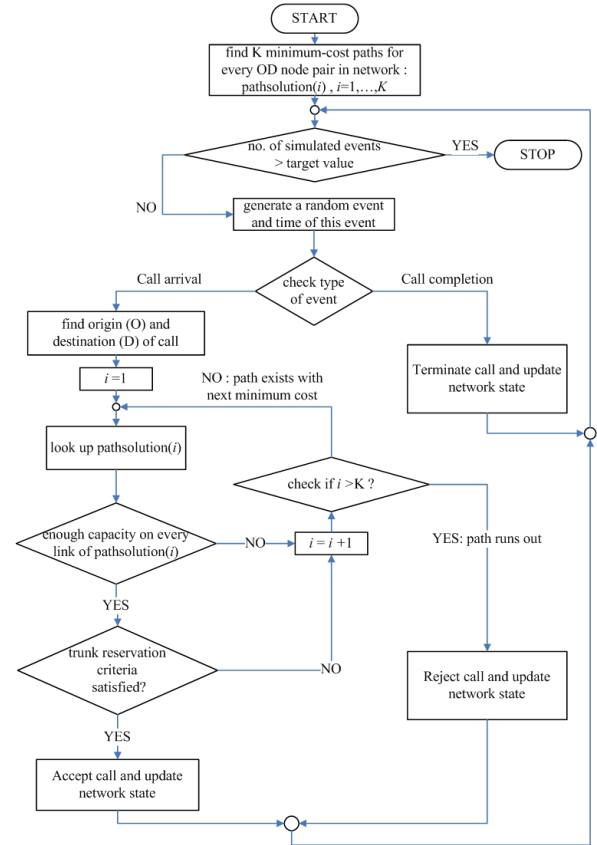

Figure 3: Computer simulation flowchart of unicast traffic.

NGN performance analysis module assumes the usage of sequential routing [9], whereby paths are tried in sequence according to their increasingly sorted costs. Furthermore, in the NGN design module, a call is allowed to use any portion of link capacity for its access. This is called complete sharing policy of call admission control (CAC). However, it is noted that engineers may want to execute $\mathrm{CAC}$ in a more controllable way. For instance, demands may be granted access to network resources in their respective order of priority. Therefore, in the NGN performance analysis module, we have adopted the well-known trunk reservation policy for CAC [10], [11]. That is, a call will be accepted into a link only when the remaining capacity on that link after accepting the call remains greater than a threshold called trunk reservation parameter.

Both sequential routing and trunk reservation features have been integrated into the discrete-event simulation (e.g. [12]) in the NGN performance analysis module. For supporting the performance analysis in NGN, simulation processes of unicast and multicast traffics are performed as follows.

\subsection{Simulation Process of Unicast Traffic}

Calls between each origin-destination node pair are allowed on only one minimum-cost path. If that path is not available at the call arrival time, then that call must be blocked and lost immediately. However, by implementing sequential routing and trunk reservation concepts, the routing table can often provide alternatives on path selection. That is, if the path with minimum cost is not available, then the candidate paths with next minimum costs should be given a trial. Fig. 3 gives a process of unicast traffic simulation.

\subsection{Simulation Process of Multicast Traffic}

With the same idea as for unicast traffic case, the sequential routing and trunk reservation are also applied in the acceptance process of multicast call session. For every VPN with multiple multicastcall sessions, every call session constructed by a single ingress node and multiple egress nodes is routed on its own minimum-cost span- 


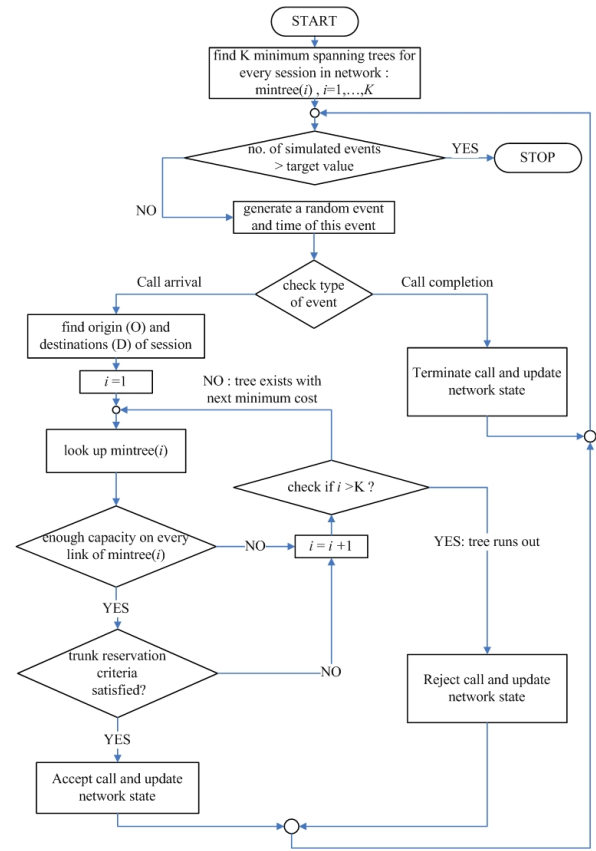

Figure 4: Computer simulation flowchart of multicast traffic.

ning tree. However, if there is a single link on that tree which is not available at a call arrival time, then the next minimum-cost candidate tree with sufficient capacity level will be selected. A process of multicast traffic simulation is shown in Fig. 4.

Due to the complete partitioning scheme of VPNs, engineers can execute simulation module and analyse each of the VPNs separately. The NGN software has been implemented by using Java running on the Red hat LINUX operating system. It is then very convenient for engineers to execute our software in simultaneous processes, each is responsible for the performance analysis of individual VPNs.

\section{NUMERICAL EXAMPLES}

To demonstrate the NGN software, we have chosen a particular network design and analysis scenario based on a modified version of a company's core network which spans across the whole country of Thailand. Fig. 5 depicts the topology and link cost (per capacity unit) of the chosen network example. The capability of NGN software implementation is tested in two scenarios as follows.

\subsection{Scenario 1: Network Design for Unicast Traffic}

In this scenario, the aim is to demonstrate the NGN software in designing a network with unicast traffics. In the test, the traffic intensity is set to 100 call Erlangs for every node pair. The call service is the standard IP-based VDO phone with equivalent capacity function parameters $b=0.12 \mathrm{~s}, \rho=0.25, R_{\text {peak }}=0.768 \mathrm{Mbps}, x$ $=15 \mathrm{kBytes}, P S_{a}=1.5 \mathrm{kBytes}$ and average call holding time $=30$ mins. The QoS targets are set to $C B P_{t}=0.3, P L_{t}=0.001$ and $P D_{t}=0.4 \mathrm{~s}$. Fig. 6 presents the result of link capacity computation from the NGN design module and Fig. 7 shows that the design target in terms of call blocking probability is guaranteed on every link. The output from design module shows that the optimal network is reached by minimum-cost path assignment.

From the functional topology in Fig. 6, we further analysed the performance of network by invoking the NGN performance analy-

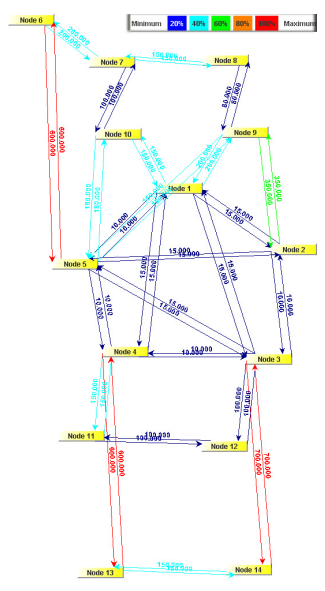

Figure 5: Topology and link cost of example network.

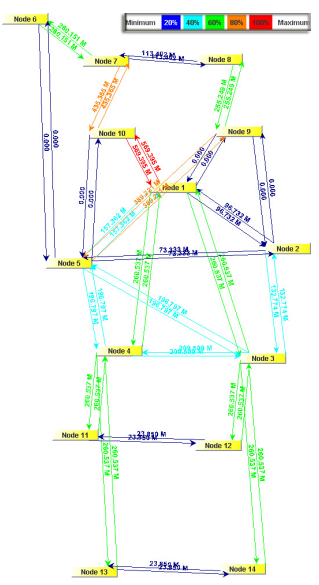

Figure 6: Link capacity assignment for unicast traffic from NGN design module.

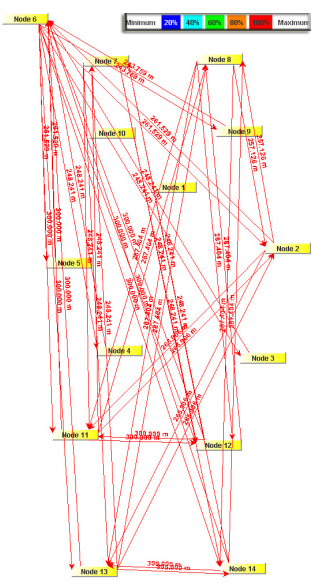

Figure 7: Call blocking probability between each OD from NGN design module: displayed only OD pairs with high call blocking probabilities. 


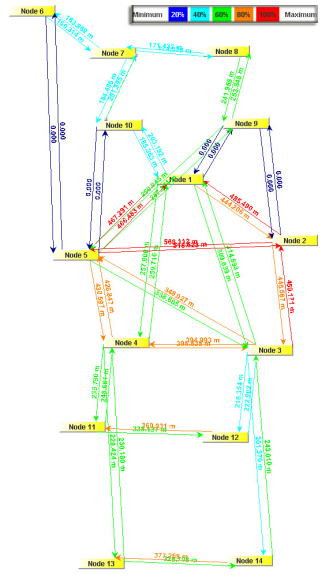

Figure 8: Call blocking probability on each link from NGN performance analysis module.

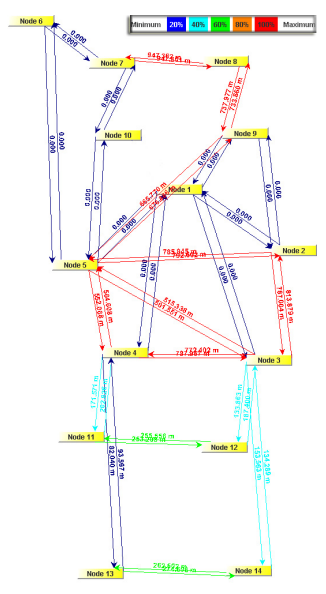

Figure 9: Call blocking probability on each link from NGN performance analysis module in the case of link failure.

sis module. The resultant call blocking probability from the NGN performance analysis module is depicted in Fig. 8. Here, we set the maximum number of candidate routes to 5 and the trunk reservation to 80 percent of link capacity. Note that all the bottleneck links with high level of call blocking probability can be easily visualised by our GUI. Additionally, it should also be noted here that other performance and design parameters can also be visualised conveniently via our developed GUI.

Apart from this network example, we have also tried out experiments on various situations. For instance, we can adopt the NGN software to study the effect of link failure, traffic surge, rerouting strategy, upgraded link capacity and predicting the network bottlenecks. A case study of given link failure scenario have been examined. Fig. 9 depicts a significant increase in call blocking probability spreading over several links when links between node 1 and node 10 have failed. With this information, a plan on backup link capacity can be included, as shown in Fig. 10. As a result, a network planer can learn to invest on upgrading network equipment based on the criticality of different parts in the network.

\subsection{Scenario 2: Network Design for Multicast Traffic}

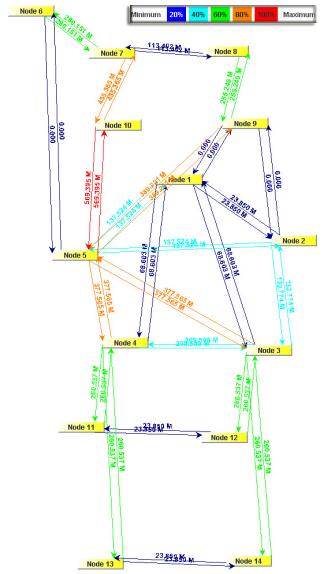

Figure 10: Link capacity assignment from NGN design module in the case of link failure.

Table 1: Multicast sessions

\begin{tabular}{|c|c|c|}
\hline Session no. & Origin & Destination \\
\hline 1 & node5 & node2, node3, node9, node14 \\
2 & node1 & node2, node5, node9 \\
3 & node3 & node11, node13, node14 \\
4 & node10 & node1, node4, node6 \\
5 & node14 & node7, node10, node12, node13 \\
\hline
\end{tabular}

Consider a network with both unicast and multicast traffics. We use the same settings for unicast traffic as in Scenario 2. For multicast traffic, the traffic intensity is set to 200 call Erlangs for every session. The call sessions are shown in Table 1 .

Fig. 11 presents the result of link capacity computation from the NGN design module and Fig. 12 guarantees that the design target in terms of call blocking probability has been well met. Then, from the link capacity assignment in Fig. 11, the performance in terms of call blocking probability is illustrated in Fig. 13. As suggested by the obtained results, the NGN software can be applied to design the network with heterogenous traffic in NGN. It is believed that the NGN software herein developed can be a useful tool for traffic engineering tasks of network engineers responsible for the wellbeings of NGN.

\section{CONCLUSION}

This paper has summarised our experiences in developing the NGN design and analysis software, which is the result of collaborative efforts between academic researchers in the university and the practical network engineers in the industry. This software has been designed on the foundation of traffic engineering. It consists of three main modules, namely, NGN design module, NGN performance analysis module and GUI module. In the NGN design module, the design objective is to minimise the overall network cost needed to provide a specified set of traffic demands with their desired QoS targets. An automatic NGN design algorithm has been given for the unicast and multicast service provisions, based on the complete partitioning scheme of VPNs. In the NGN performance analysis module, the objective is to analyse the QoS parameters in both the time scale of call and packet dynamics (e.g. call blocking probability, packet loss probability, average packet delay). This module relies on the technique of discrete-event computer simu- 


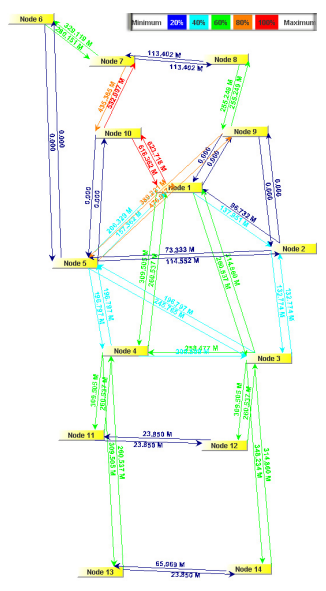

Figure 11: Link capacity assignment for multicast traffic from NGN design module.

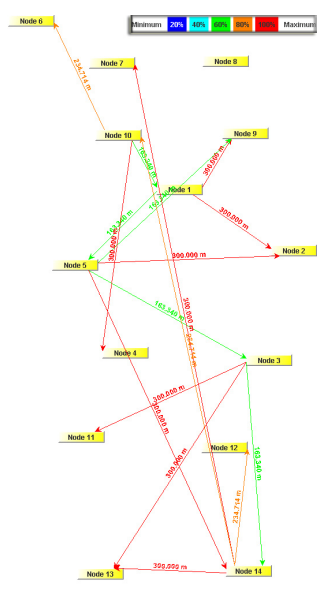

Figure 12: Call blocking probability between each OD from NGN design module.

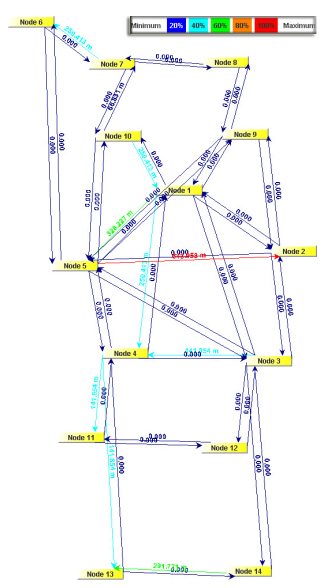

Figure 13: Call blocking probability on each link from NGN performance analysis module. lation, where the standard sequential routing and trunk reservation CAC have been emulated. Preliminary results from both the NGN design and performance analysis modules have been given to demonstrate the software capability in dealing with the real-world network size. Due to the involved complexities, it is recommended that the platform for running the NGN software should be workstation class. In our project, we have simultaneously utilised two workstation machines, each with dual $2.8 \mathrm{GHz}$ Xeon CPU, 2 GB RAM. Finally, all input and output parameters can be interfaced via the developed GUI, which has been specifically designed in our software based on feedbacks from practical network engineers who have experimented up with our software. Therefore, for those directly responsible for planning the NGN platforms, it is easy to use and understand the developed GUI in our software. Therefore, GUI in our software can be easy to use and understand by those directly responsible for planning the real NGN platforms.

\section{Acknowledgment}

The authors would like to acknowledge the suggestions in the NGN project from Dr Anotai S., Dr Anak M. and engineers from CAT Telecom PLC, Thailand. The authors would also like to acknowledge all the efforts of every member of (NG) ${ }^{2}$ (Next Generation Network Group) at Chulalongkorn University, Thailand.

\section{REFERENCES}

[1] ITU-T Recommendation Y. 2261. PSTN/ISDN Evolution to NGN, September 2006.

[2] ITU. A Handbook on Internet Protocol (IP)-Based Networks and Related Topics and Issues, 2005.

[3] www.opnet.com

[4] www.vpisystems.com

[5] C. Aswakul, et. al. Development of Computer Simulation Software for Design and Analysis of Next Generation Telecommunication Network. Final Report Submitted to the Research and Development Department, CAT Telecom PLC, Thailand, 2007.

[6] K. W. Ross. Multiservice Loss Models for Broadband Telecommunication Networks. Springer Verlag, 1995.

[7] R. Guerin, H. Ahmadi and M. Naghshineh. Equivalent Capacity and Its Application to Bandwidth Allocation in High-Speed Networks. IEEE Journal on Selected Areas in Communication, 9(7):969-981,1991.

[8] D. Bertsekas and R. Gallager. Data Networks. 2nd Ed., Prentice Hall, 1992.

[9] A. Girard. Routing and Dimensioning in Circuit-Switched Networks. Addison-Wesley, 1990.

[10] J. W. Roberts. Teletraffic Models for the Telecom I Integrated Services Network. In Proceedings of 10th International Teletraffic Conference, paper 1.1-2 Montreal, Canada, 1983.

[11] C. Aswakul and J. Barria. Analysis of dynamic service separation with trunk reservation policy. IEE Proceedings Communications, 149(1):23-28, February 2002, .

[12] A. M. Law and W. D. Kelton. Simulation Modeling and Analysis, 3rd Ed., McGraw-Hill, 2000.

[13] H. Yamada. End-to-end performance design framework of MPLS virtual private network service across autonomous system boundaries. In Proceedings of Networks 2006, 2006.

[14] R. Hwang, et al. Multicast routing based on genetic algorithns. Journal of Information Science and Engineering, 16:885-901, 2000. 